\title{
The relationships between rugby union, and health and well-being: a scoping review
}

\author{
Steffan A Griffin ำ 1,2 Nirmala Kanthi Panagodage Perera 두 , 3,4 \\ Andrew Murray (1) ${ }^{1,5}$ Catherine Hartley ${ }^{0}{ }^{6},{ }^{6}$ Samantha G Fawkner, ${ }^{7}$ \\ Simon P T Kemp (1), 2,8 Keith A Stokes 주, 2,9 Paul Kelly (i) ${ }^{10}$
}

${ }^{1}$ Centre for Sport and Exercise, The University of Edinburgh, Edinburgh, UK

${ }^{2}$ Medical Services, Rugby

Football Union, London, UK ${ }^{3}$ Centre for Sport, Exercise and Osteoarthritis Research Versus Arthritis, Botnar Research Centre, University of Oxford, Oxford, UK

${ }^{4}$ Sports Medicine, Australian Institute of Sport, Bruce, Australian Capital Territory, Australia

${ }^{5}$ Scottish Rugby Union,

Edinburgh, UK

${ }^{6}$ Bodleian Health Care Libraries,

Oxford, UK

${ }^{7}$ The Institute for Sport, Physical Education and Health Sciences, The University of Edinburgh, Edinburgh, UK

${ }^{8}$ Faculty of Epidemiology and Public Health, London School of Hygiene \& Tropical Medicine, London, UK

${ }^{9}$ Department for Health, University of Bath, Bath, UK ${ }^{10}$ Physical Activity for Health Research Centre, The University of Edinburgh, Edinburgh, UK

\section{Correspondence to} Dr Steffan A Griffin, Centre for Sport and Exercise, The University of Edinburgh, Edinburgh EH8 9YL, UK; steffangriffin@gmail.com

Accepted 17 September 2020 Published Online First 28 October 2020

\section{ABSTRACT}

Objective To scope the relationships between rugby union, and health and well-being.

Design Scoping review.

Data sources Published and unpublished reports of any age, identified by searching electronic databases, platforms and reference lists.

Methods A three-step search strategy identified relevant published primary, secondary studies and grey literature, which were screened using a priori inclusion criteria. Data were extracted using a standardised tool, to form (1) a numerical analysis and (2) a thematic summary.

Results and discussion 6658 records were identified, and 198 studies met the inclusion criteria. All forms of rugby union can provide health-enhancing physical activity (PA). 'Non-contact' and wheelchair rugby in particular provide a wide range of physical and mental health and well-being benefits. The evidence is either mixed or unclear in relation to 'contact' rugby union and its effects on a range of physical health domains. Injury and concussion incidence rates are high for contact rugby union relative to other sports.

Conclusions A wide range of stakeholders as well as existing and potential participants can use this information to make a more informed decision about participating in and promoting rugby union as a healthenhancing activity. Industry and policy-makers can use this review to inform policies and strategies that look to increase participation rates and use rugby union as a vehicle to contribute positively to population health. Further research understanding rugby union's contribution to PA as well as to muscle-strengthening and balance is indicated, as well as research examining more health and well-being outcomes across more diverse cohorts.

\section{INTRODUCTION}

Rugby union is a team-based sport, played in over 120 countries by over 8 million people. ${ }^{1}$ Multiple forms of the game exist, with different rules as to the number of players on each side (traditionally 15 or 7), and the degree of contact involved (full contact/non-contact e.g. 'tag' touch'). There are also adapted forms of rugby union for athletes with disabilities, such as wheelchair rugby.

As with many team and individual sports, any form of rugby union can involve moderate-tovigorous physical activity (MVPA), ${ }^{2}$ which alongside being 'non-sedentary' confers a wide range of physical, mental and social well-being benefits. ${ }^{3-6}$
Indeed, there are widely published health benefits associated with participating in various individual and team sports, ${ }^{7} 8$ including improved aerobic fitness, cardiovascular function, metabolic fitness and in some cases reduced mortality. ${ }^{9}$ Sport is also considered an 'underused yet important' contributor to physical activity (PA) and health ${ }^{10}$ in the World Health Organisation's (WHO) 2019 Global Action Plan for Physical Activity.

Despite global participation in rugby union, there has not been an overarching review of the evidence on the specific relationships between rugby union, and health and well-being. A systematic review of the potential health benefits of different sporting disciplines $^{7}$ found 69 studies in total, but only 1 focused on rugby union. Scientific efforts to date have focused mainly on the relationship between rugby union participation and injury. However, given that health (as per WHO definition) is not 'merely the absence of disease or infirmity', ${ }^{11}$ there is a need to consider the potential impact of rugby union participation on health and wellbeing. Existing as well as potential future participants and various other stakeholders (eg, parents, medical professionals, schools, clubs and unions) need to know the likely benefits as well as the risks to make an informed choice about rugby union participation. $^{12}$

Therefore, and in line with this approach being used in different sports, ${ }^{13}$ the aim of this scoping review is to inform participation, policy, advocacy and research strategy by:

(1) Identifying the relationships between rugby union and various health and well-being outcomes.

(2) Identifying evidence gaps and key research priorities for rugby union and health and well-being.

\section{METHODS}

A scoping review was considered the most appropriate methodological approach to address this aim, especially given the iterative nature of researching what is a novel topic. Scoping reviews are a type of review that seek to identify the nature and extent of research evidence for a given topic, in comparison to, for example, systematic reviews that seek to provide an exhaustive appraisal and synthesis of research evidence. ${ }^{14}$ Scoping reviews are, therefore, characterised as broad in nature, and are often focused on mapping key concepts that underpin research areas, and detailing the range of evidence available for a certain topic. ${ }^{15-17}$

This scoping review is based on Arksey and O'Malley's five-stage scoping review process, and 


\begin{tabular}{|c|c|}
\hline Inclusion criteria & Exclusion criteria \\
\hline $\begin{array}{l}\text { Research articles not limited by age of study, by } \\
\text { geographical location, language or setting. } \\
\text { Research articles that consider all abilities, } \\
\text { age groups and gender of participants, as well } \\
\text { as specific population groups (with a specific } \\
\text { health condition). } \\
\text { Research articles that consider all forms of } \\
\text { rugby union (eg, } 7 \text {-a-side/10-a-side, 15-a-side/ } \\
\text { disability/touch/tag/beach/veterans/rugbyX). } \\
\text { Sources of information including primary } \\
\text { research studies, reviews, systematic reviews, } \\
\text { scoping reviews, meta-analyses, guidelines, as } \\
\text { well as grey literature to include unpublished } \\
\text { and ongoing trials, annual reports, dissertations } \\
\text { and conference proceedings. } \\
\text { Outcome variables that must be health related } \\
\text { in terms of: physical activity (eg, metabolic } \\
\text { equivalent of task value), physiological demand, } \\
\text { mortality, morbidity or disease risk factors (eg, } \\
\text { effect on blood pressure); cardiorespiratory, } \\
\text { neurological, metabolic or musculoskeletal } \\
\text { function/fitness; or social/psychological and } \\
\text { more holistic measures of 'well-being'. } \\
\text { Systematic reviews in which the outcome } \\
\text { variables were injuries or other acute health } \\
\text { issues. }\end{array}$ & $\begin{array}{l}\text { Anthropometric/biomechanical } \\
\text { studies or studies relating to } \\
\text { performance, recovery or injury } \\
\text { prevention. } \\
\text { Opinion pieces/opinions, } \\
\text { magazine and newspaper } \\
\text { articles. } \\
\text { Research articles that do } \\
\text { not scientifically investigate } \\
\text { measures of health, or directly } \\
\text { relate to function, or fitness (or } \\
\text { do not include data). } \\
\text { Cadaver studies. } \\
\text { Health and safety/occupational } \\
\text { issues not related to playing or } \\
\text { watching rugby union. } \\
\text { 'Contact' rugby league studies. } \\
\text { Research articles in which the } \\
\text { outcome variables were injuries } \\
\text { or other acute health issues that } \\
\text { are not systematic reviews or } \\
\text { meta-analyses. }\end{array}$ \\
\hline
\end{tabular}

informed by Levac et al's framework, as well as the work of the Joanna Briggs Institute and previously published relevant scoping reviews. ${ }^{13}$ 15-18 The protocol was published in an openaccess journal. ${ }^{19}$ See online supplementary appendix 1 for the completed Preferred Reporting Items for Systematic Reviews and Meta-Analyses (PRISMA)-Scoping Review (ScR) checklist. ${ }^{20}$

\section{Stage 1: identify the research question(s)}

Following a preliminary literature search and discussions between the author groups, the following research questions were developed to reflect the population, context and content of the review.

- What is known about the relationships between rugby union, and health and well-being?

- What are the evidence gaps in the field?

-What are the key research priorities in the field?

\section{Stage 2: identifying relevant studies}

The a priori inclusion and exclusion criteria (table 1) were determined based on existing frameworks and discussions within the research group. ${ }^{13} 18$ Essentially, we wanted this scoping review to include a broad range of studies that included participants of any population involved in rugby union, in any capacity, with studies not limited by factors such as age or language. Owing to the iterative nature of the scoping review, important elements of our methods should be noted.

A decision was made to limit studies to those referring to 'rugby union' only, and to report on the outcomes by the type of rugby union referred to in the studies (contact/non-contact/ wheelchair). Following author group discussion, it was decided that given at a practical level, non-contact forms of rugby union and rugby league are identical and played under the same rules, these would be treated as the same, and as such studies relating to 'non-contact' forms of rugby league would be included in the analysis. We did not extend this principle to 'contact' forms of rugby league for several reasons. First, our initial limited search returned a volume of material for both rugby league and rugby union that was beyond capacity for this scoping study. Second, in regard to contact forms of rugby league and rugby union, there are inherently different rules, patterns of play and demands between both sports that limit comparability.

In regard to injuries, given the volume of research, and focus in this area, a decision was made to limit injury studies to systematic reviews (as per the published protocol). ${ }^{19}$ This has allowed us to report review-level evidence on this topic, using previous syntheses conducted by other author teams, as has been done before in different subject areas. ${ }^{21}$ One potential approach considered was to exclude studies in which the outcome variables are injuries or other acute health problems (as per previously published systematic reviews). ${ }^{7}$ However, we decided that this approach might overlook a key concern that the author group's professional experience tells us is critical to stakeholders, and would limit the applicability of this scoping review.

\section{Search strategy}

The three-step search strategy (an initial limited search, followed by the identification of key words and index terms, and then further searching of references and citations) using eight research databases is summarised in online supplementary appendix 2. The eight databases searched included: Medline, Embase, PsycINFO, SPORTDiscus, Web of Science Core Collection, Google Scholar, ProQuest Dissertations \& Theses Global and WHO-International Clinical Trials Registry Platform.

\section{Stage 3: study selection}

Relevant titles and abstracts identified by the search strategy were evaluated against the eligibility criteria by the lead author (SAG). NKPP subsequently completed the same process on a random sample of $20 \%$ of titles and abstracts, using the same inclusion and exclusion criteria, with 97\% overall concordance (defined as percentage positive agreement between authors). SAG then reviewed the titles and abstracts of all other studies and sourced the full text for all articles meeting the inclusion criteria. Authors were contacted where full texts were not available.

\section{Stage 4: charting the data}

SAG and NKPP designed a data extraction form (see online supplementary appendix 3). SAG and NKPP trialled the data extraction form on 5 randomly selected studies, and independently extracted data from the first 10 studies to determine whether their approach to data extraction was consistent with the research question and purpose. Following this step, SAG extracted data from $90 \%$ of included studies and NKPP extracted data from $10 \%$ of studies. SAG checked $10 \%$ of NKPP's data extractions for accuracy and vice versa, ${ }^{19}$ with $96 \%$ overall concordance. As per a previously published scoping review in a relevant field, no further cross-checking was performed given the high level of concordance. ${ }^{13} 18$

\section{Stage 5: collating, summarising and reporting the results}

Data are presented in the following formats:

1. Numerically, alongside a scoping review flowchart. The data are presented by theme (see description below), period of publication, country of origin and study type.

2. Thematically, using a descriptive analysis to map key findings and concepts relevant to the research question. SAG used an inductive thematic analysis framework, and based the analysis on Braun and Clarke's six-phase framework. ${ }^{22}$ Familiarisation with the data was achieved by manually extracting key data from individual studies. These were then individually categorised according to the primary focus 


\begin{tabular}{|c|c|c|c|c|c|}
\hline 1 Initial Themes: & Physical Demand & Physical Health \& Function & Mental Health & Wellbeing & Acute Health Issues \\
\hline \multirow[t]{9}{*}{ Coded Extracts } & Non-sedentary time & Cardiovascular health \& function & Psychological health & Wheelchair Rugby & Concussion \\
\hline & Physical Activity & Metabolic health \& function & Mood & Activities of daily living & Injuries \\
\hline & Distance covered & Musculoskeletal Health (OA) & Enjoyment & Social benefits & \\
\hline & Cardiovascular stress & Bone mass density & & Life satisfaction & \\
\hline & Physical demands & Neurological health \& function & & Identity & \\
\hline & Metabolic demand & Immunological health \& function & & Independence & \\
\hline & & Respiratory health \& function & & Quality of life & \\
\hline & & Body composition & & Academic achievement & \\
\hline & & Longevity & & Employment & \\
\hline Refined Themes: & Physical Demand & Physical Health \& Function & Mental Health & Wellbeing & Acute Health Issues \\
\hline \multirow[t]{7}{*}{ Refined Sub-Themes } & Activity Profile (Physical Activity + Non-Sedentary Time + Distance Covered + Physical Demands) & Longevity & & Wheelchair Rugby & Injuries \\
\hline & Cardiovascular \& Metabolic Demand & Cardiovascular health \& function & & Other forms of rugby & Concussion \\
\hline & & \multicolumn{2}{|c|}{ Metabolic health \& function (inc body composition) } & & \\
\hline & & Neurological health \& function & & & \\
\hline & & Respiratory health \& function & & & \\
\hline & & Immunological health \& function & & & \\
\hline & & Musculoskeletal health \& function & $(B M D+O A)$ & & \\
\hline
\end{tabular}

Figure 1 A summary of how themes were generated, based on Braun and Clarke's six-phase framework. *OA (osteoarthritis)

and main outcome measures. These categories were then grouped to form themes, which were subsequently reviewed and refined to include subthemes where appropriate. ${ }^{162223} \mathrm{~A}$ visual summary of this process is provided in figure 1 . Where a vast number of studies were assigned to a specific theme (as can be seen in online supplementary appendix 4) priority would be given to reporting the results from studies that the authors believe are most relevant to some of the aforementioned stakeholders and policy-makers, or where more 'gold-standard' outcome measures were used, or where certain patterns were clear from the data. In cases where individual studies are highlighted, we have also tried to highlight any methodological issue(s) associated with them that may impact the real-world applicability of the data.

\section{(Optional) stage 6: consultation}

The author group have been involved with the sport of rugby union in many different forms. We authors have played, coached and been responsible for the medical care of professional rugby union players. Similarly, there is extensive experience within the group in not only conducting health and well-being research specific to rugby, but also in sport, physical activity and public health in general. Multiple members of the group are also employed by and advise the board of two of the UK's national rugby governing bodies (Rugby Football Union and Scottish Rugby Unionn (RFU and SRU)), as well as have roles in advising

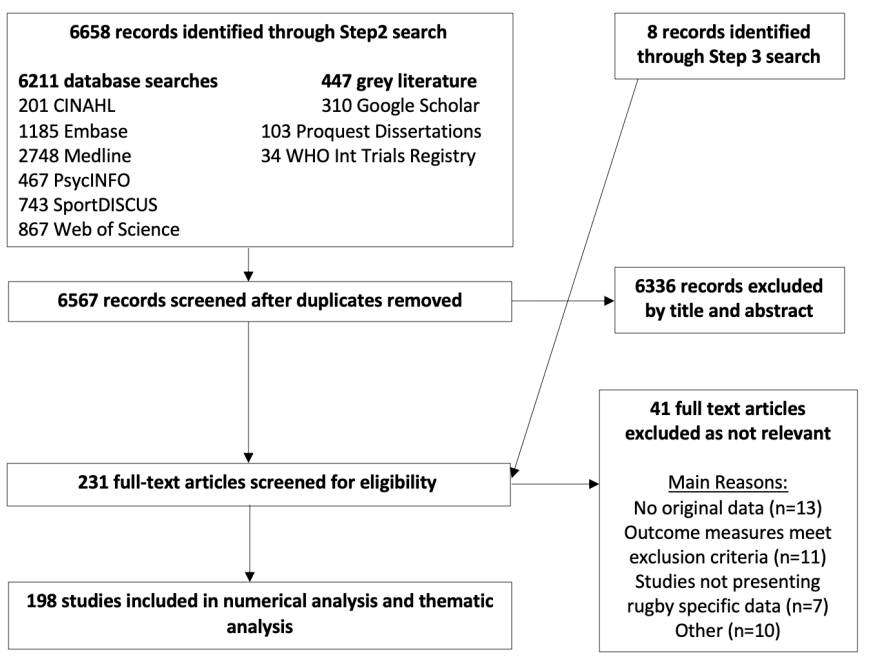

Figure 2 Scoping review study selection flowchart. about physical activity and sport at a governmental level. Given the breadth of knowledge and experience within the group in working with key stakeholders as such, we did not host an external consultation exercise, though we will be involving additional groups in both the dissemination phase of this project, and in future studies (which are currently in the planning process).

\section{RESULTS}

Numerical analysis

The initial search identified 6658 studies, 6211 of which came from recognised databases and 447 from grey literature. Six thousand five hundred and sixty-seven studies remained after further duplicates were manually removed from the search. Six thousand three hundred and thirty-six studies did not meet the inclusion criteria. Of the 231 identified studies, 41 were deemed irrelevant and removed following full text review (with reasons provided in the flowchart-figure 2). Eight additional studies were included following further searches of references and author consultation. Overall, 198 studies met inclusion criteria and were included in the analysis. The scoping review flowchart details the search and study selection process (figure 2).

\section{Chronology and geography of included studies}

Figure 3 highlights the substantial chronological increase in the number of related studies, with a rate of increase greater than that seen in the only comparable review in golf and health. ${ }^{13}$ Geographically, table 2 demonstrates the contribution per continent, and the country in each continent with the largest contribution to the literature. Europe provided the majority of included studies (47\%), and the UK was the main contributor within this region ( $\mathrm{n}=41$ studies). Australasia (16\%), North America (15\%) and Africa (11\%) were other major contributors. South Africa contributed all of the included studies within the African continent. Asia (7\%) and South America (5\%) provided a low proportion of the overall included studies, in keeping with the recognition that these are generally considered less established rugby union markets. ${ }^{24}$ All of the 198 final studies were published in English, despite the sizeable contribution from a diverse range of countries where English would not be considered the native language.

Type of studies

Table 3 details the type of studies included in the review.

Themes identified

Studies fell within five broad themes: 


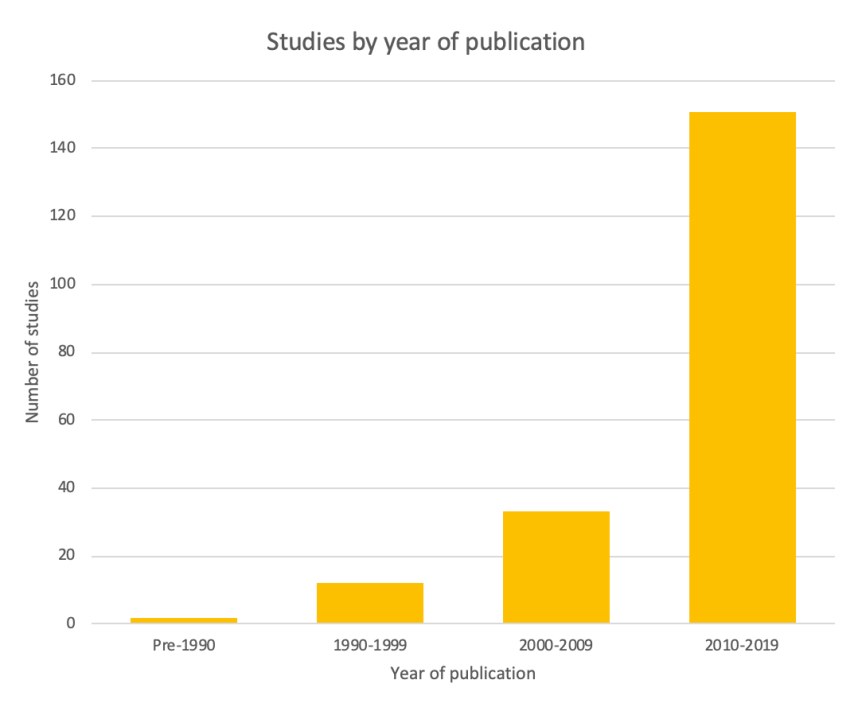

Figure 3 Included studies by year of publication.

Rugby union and physical activity $(\mathrm{n}=51)$.

Rugby union, physical health and function $(n=95)$.

Rugby union, mental health and function $(n=11)$.

Rugby union and well-being $(n=21)$.

Systematic reviews of rugby union injuries or acute health issues $(n=20)$.

\section{Thematic analysis}

Theme 1: rugby union and physical activity

Fifty-one studies reported on the level/volume/amount of PA conducted while playing rugby union across different levels and formats of the game. The majority of this research was performed in men's rugby union, with a relative lack of research breadth and depth in women's rugby union across all formats of the game ( $70 \%$ vs $14 \%$ of studies respectively). Most research focused on adult (18 years and older) rugby union, with $12 \%$ of studies primarily involving youth athletes.

Contact rugby union (15-a-side (15s), 7-a-side (7s), etc)

Data from one study (with ambiguity over participant number, gender and analysis) suggests that traditional 15 s rugby union has a mean metabolic equivalent of task (MET) value of 8.3 (8.1-8.5), ${ }^{2}$ classifying it as a vigorous form of PA. Various crosssectional studies also show that training and matches in $15 \mathrm{~s}$ and $7 \mathrm{~s}$ (across both men and women's rugby union) provide a strenuous cardiovascular stimulus, ${ }^{25-28}$ with $7 \mathrm{~s}$ matches (which are shorter in duration than $15 \mathrm{~s}$ matches) providing a more intense cardiovascular stimulus than $15 \mathrm{~s}^{29}$

\begin{tabular}{lll}
\hline Table 2 & Geography of included studies & \\
\hline Continent & $\begin{array}{l}\text { Number of } \\
\text { included studies }\end{array}$ & $\begin{array}{l}\text { Country with most included } \\
\text { studies (and number of } \\
\text { included studies) }\end{array}$ \\
\hline Europe & 93 & UK $^{41}$ \\
\hline Australasia & 31 & Australia $^{19}$ \\
\hline North America & 29 & USA $^{18}$ \\
\hline Africa & 21 & South Africa $^{21}$ \\
Asia & 14 & Japan $^{6}$ \\
\hline South America & 9 & Brazil $^{5}$ \\
\hline Not specified & 1 & Not specified \\
\hline
\end{tabular}

Table 3 Type of studies included

\begin{tabular}{ll}
\hline Type of study & Number of included studies \\
\hline Systematic reviews & $22(11 \%)$ \\
\hline Randomised control trials & $5(2.5 \%)$ \\
Cross-sectional studies & $118(59.5 \%)$ \\
Cohort studies & $43(22 \%)$ \\
Qualitative studies & $5(2.5 \%)$ \\
Other & $5(2.5 \%): 1$ thesis, 1 non-randomised controlled trial, \\
& $\begin{array}{l}\text { 2 non-systematic 'review' articles and 1 mixed case } \\
\text { report and non-systematic review }\end{array}$ \\
\hline
\end{tabular}

Non-contact rugby (touch, tag, etc)

Cross-sectional data from a population of 101 male and female 11-14 year olds indicates that touch and tag rugby have MET values of $6.3(5.6-7.2)^{2}$ and $6.0(4.5-7.5),{ }^{30}$ respectively, which correspond to vigorous-intensity PA. A cross-sectional study of 16 male touch rugby players showed that they spent approximately $75 \%$ of time at over $80 \%$ of maximal heart rate (HRmax) during match play. ${ }^{31}$

\section{Wheelchair rugby}

Wheelchair rugby has a MET value of 3.8-4.3 (dependent on position) according to a cross-sectional study involving 52 male participants ${ }^{32}$. This would correspond to moderate-intensity PA using able-bodied cut-off scores, though the relative intensity may be different in this population relative to the parameters generally promoted in able-bodied populations. Wheelchair rugby players have also been shown to spend up to half of match play at above $90 \%$ of HRmax, which the authors of the paper suggest is sufficient to generate improvements in cardiorespiratory parameters in this population. ${ }^{33}$

The MET values for the various forms of rugby union are shown below in figure 4 .

The majority of studies $(n=32)$ described the movement profile (eg, distance covered, time spent moving) associated with various forms of rugby union, with all showing that rugby union participation results in important contributions to these indices of PA. ${ }^{25} 28$ 34-63 This review did not find any studies that explored an association between rugby union participation and muscle-strengthening and balance improvement. National population surveys, however, typically 'count' rugby union as an activity that can provide muscle-strengthening/balance improvement when accrued for period of over $10 \mathrm{~min} .{ }^{64-67}$ There is also

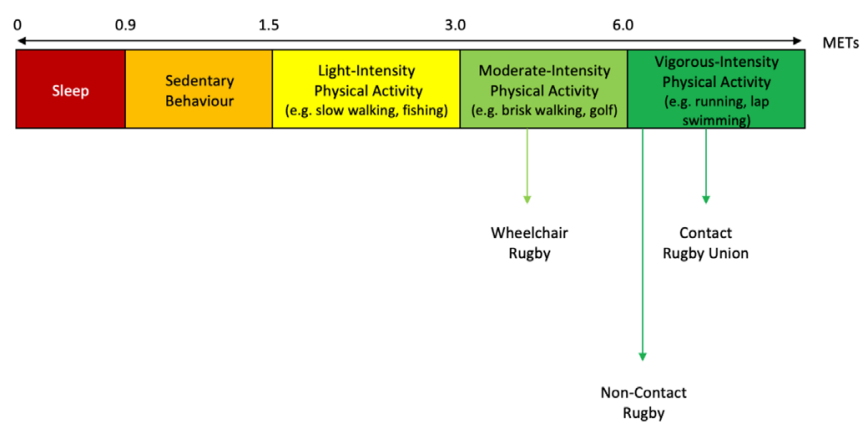

Figure 4 MET values attributed to different forms of rugby union. Light-intensity activities are defined as 1.1 METs to 2.9 METs, moderateintensity activities are defined as 3.0-5.9 METs, and vigorous-intensity activities are defined as 6.0 METs or more. *MET (metabolic equivalent of task) 
non-rugby union-specific evidence that 'ball sports' can improve muscle function, bone health and balance. ${ }^{68} 69$

Theme 2: rugby union, physical health and function Contact rugby union (15s, $7 \mathrm{~s}$, etc)

\section{Cardiovascular health and function}

One cross-sectional study comparing a cohort of 259 retired elite rugby union players to the general population (using data from the English Longitudinal Study of Aging (ELSA)), found that the incidence of high blood pressure was significantly lower in former rugby players when adjusted for age. ${ }^{70}$ This is consistent with another cross-sectional study among 52 retired international rugby union players, that reported a lower frequency of chronic cardiovascular disorders in this cohort relative to controls (matched for both age and social deprivation). ${ }^{71}$ This evidence base is contrasted somewhat by the results of crosssectional studies among over 200 veteran rugby union players from 10 countries, that demonstrated a higher incidence of hyperlipidaemia and hypertension compared with the Australian general population. ${ }^{72-74}$ However, it is not clear whether these cohorts were consistently matched in terms of age and gender, or measures put in place to account for the significantly higher relative body mass index among veteran rugby union players, which might skew the results. ${ }^{73}$ Similar contrasting findings exist for the effect of rugby union on lipid profile. ${ }^{75-79}$

\section{Metabolic health and function}

The relationship between rugby union and metabolic health and function is similarly unclear. In standardised morbidity ratio analyses of 259 retired elite male players aged over 50 years, diabetes rates were significantly lower compared with ELSA participants. ${ }^{70}$ Again this is in contrast to the higher metabolic risks reported among the previously mentioned cohort of veteran rugby union players, who had a higher incidence of diabetes relative to an age-matched Australian control group. ${ }^{73}$

\section{Musculoskeletal health and function}

The prevalence of osteoarthritis and rates of joint replacements are significantly higher among both former elite players ${ }^{80} 81$ and former amateur players ${ }^{82}$ relative to the general population. Data suggests an association between rugby union participation and increased bone mass density (BMD), especially when compared with both sedentary controls and athletes from other sports. ${ }^{83-90}$ Both cross-sectional and longitudinal epidemiological studies, however, have shown that increased BMD may be considered as part of the osteoarthritis spectrum, ${ }^{91}$ and one cross-sectional study reported a significant increase in osteoporosis among a retired elite male cohort. ${ }^{70}$ One area of particular academic interest is degenerative lesions of the cervical spine (especially among front-row rugby union players). ${ }^{92-96}$ While all studies reported premature and advanced changes of degenerative disease, these changes did not always manifest themselves clinically or affect activities of daily living. ${ }^{93} 95$

\section{Neurological health and function}

Research on potential neurological sequelae comprised nearly a third of the studies categorised under the 'physical health' theme (31\%). Overall, there are mixed reports regarding longterm cognitive function and neurological health in retired ablebodied rugby union players. A systematic review on this topic reported normal objective neuropsychological performance in retired rugby union players across most cognitive domains (apart from fine motor control), and also that the evidence is generally both 'inconclusive and poorly developed'. ${ }^{97}$ For current players, findings are similarly mixed, with some studies reporting reduced cognitive function and cognitive vulnerability following exposure to rugby union, ${ }^{98-104}$ while other studies do not. $^{71} 105-110$

\section{Immunological health and function}

Data from a study involving 40 elite male players suggest that they have a more favourable gut microbiota relative to sedentary individuals, which is linked with a wide range of immunological health outcomes. ${ }^{111}$ However, they may be more susceptible to infection over the course of the professional season due to suppressed immunity and the sustained activation of their inflammatory response. ${ }^{112}$

\section{Non-contact rugby (touch, tag, etc)}

\section{Cardiovascular health and function}

Two small observational studies suggest that regular participation in touch rugby over a $10-12$-week period has clinically favourable effects on cardiovascular risk factors (including resting heart rate and diastolic blood pressure). ${ }^{113114}$ In one randomised control trial (RCT) of active men, the effect sizes across similar domains were greater than those reported in a group whose PA was prescribed as self-paced interval running. ${ }^{115}$ One RCT in a cohort of thirty-three sedentary men also demonstrated improvements in submaximal $\mathrm{VO}_{2}$ (rate of oxygen consumption) compared with a sedentary control group. ${ }^{116}$

\section{Metabolic health and function}

There is some evidence that suggests that participating in touch rugby improves metabolic risk factors. Using an RCT design in a cohort of 33 middle-aged inactive men, touch rugby was associated with improvements in metabolic risk factors associated with the prevention of type 2 diabetes mellitus, including $\mathrm{VO}_{2}$ at $80 \%$ HRmax, glycated haemoglobin levels and total body fat mass. ${ }^{117}$ These effect sizes were found to be similar to those provided by a continuous cycling training programme. ${ }^{117}$ Prescribing touch rugby as a mode of PA in a small $(n=10)$ sedentary male Indigenous Australian population also increased engagement in PA and assisted in the acute regulation of glucose disposal. ${ }^{118}$ Similarly, a varied activity programme that included rugby (alongside other sports, circuit-based aerobics, strength and resistance exercises) also led to an improvement in metabolic and cardiorespiratory fitness among 22 obese children. ${ }^{119}$

\section{Immunological health and function}

Data from a small $(n=9)$ RCT suggest that small-sided touch rugby games stimulate and maintain an acute anti-inflammatory response similar to that provided by stationary cycling, though there are no data to support an association between this and illness or injury risk. ${ }^{120}$

\section{Wheelchair rugby}

\section{Cardiovascular health and function}

Data from 14 high-level wheelchair rugby athletes suggest that wheelchair rugby participants have a higher aerobic capacity relative to untrained quadriplegics. ${ }^{121}$ Regularly participating in upper-body sports activities such as wheelchair rugby has also been associated with long-term reductions in carotid atherosclerosis ${ }^{122}$ and improved serum lipid profiles ${ }^{75}$ relative to sedentary controls. 


\section{Musculoskeletal health and function}

One study investigating the longitudinal effects of wheelchair rugby training on the body composition of 13 subjects with quadriplegia demonstrated that regular participation in wheelchair rugby training increased lean mass, and decreased total body fat mass. ${ }^{88}$

\section{Neurological health and function}

Data from a study comparing 19 physically active wheelchair rugby athletes at the 2015 Parapan American Games to 9 relatively inactive spinal cord injury controls suggest that physically active wheelchair rugby participants exhibit improved dynamic cerebral autoregulation and cognitive function. ${ }^{123}$

\section{Respiratory health and function}

Participation in wheelchair rugby over the course of 12 months has been linked to a reduction of respiratory dysfunction in a cohort of 10 quadriplegic athletes. ${ }^{124}$ Wheelchair rugby athletes with quadriplegia have also been shown to have enhanced respiratory muscle strength and thoracic mobility relative to sedentary quadriplegic controls (with no significant difference between levels of spinal cord injury between the two groups). ${ }^{125}$ A small $(n=15)$ group of male subjects with quadriplegia who underwent 12 months of regular wheelchair rugby training also significantly improved their pulmonary function relative to sedentary quadriplegic subjects. ${ }^{126}$

\section{Theme 3: rugby union, mental health and function}

Contact rugby union (15s, 7s, etc)

Two studies investigated symptoms of common mental disorders among retired professional rugby union players. ${ }^{127} 128$ One cross-sectional study among 295 retired professional rugby union players reported a prevalence rate of $28 \%$ for anxiety/ depression, which is generally above that reported in the general population worldwide (12\%-25\%). ${ }^{128}$ Within a current elite male rugby union playing cohort, one study of 990 players found that when matched for age and gender, the prevalence of symptoms of anxiety/depression seems slightly higher than in other general/occupational populations. ${ }^{129}$ An observational prospective cohort study in a cohort of 595 professional rugby union players reported incidence rates of symptoms of common mental disorders similar to those of professional athletes from other sports. $^{130}$

Non-contact Rugby (Touch, Tag etc)

No relevant studies found.

\section{Wheelchair rugby}

A cross-sectional study that collected data from 150 males with quadriplegia at various wheelchair rugby tournaments found that practicing wheelchair rugby 2 times or more a week (compared with once a week or less) was significantly associated with lower depressive symptomology and perceived stress. ${ }^{131}$

\section{Theme 4: rugby union and well-being}

The majority $(57 \%)$ of studies were performed in a wheelchair rugby union setting.

\section{Contact rugby union (15s, $7 \mathrm{~s}$, etc)}

A qualitative study among 15 veteran male players aged 54-83 years investigating the motivation to continue playing, reported that playing gave participants 'a sense of nostalgia and pride', 'lifelong friendship and belonging', 'pure joy and passion' and helped them be 'mentally and physically active'. ${ }^{132}$ 'Making friends' was also reported as a main motivation for playing rugby union among amateur male players aged over 35 years. ${ }^{133} \mathrm{~A}$ cross-sectional study among 339 female rugby players reported that they played rugby because 'they enjoy the game, they like the aggressive aspects of the sport, they appreciate the social aspects of the game, and they believe the sport provides them with positive benefits, such as increased fitness, confidence and strength'. 134

Three studies investigated academic achievement among adolescent rugby union players. ${ }^{135-137}$ One observational study in Ireland reported no statistically significant difference to the final Leaving Certificate Score among students who participated in rugby union. ${ }^{136} \mathrm{~A}$ retrospective analysis among 30 adolescent South African students found 'no conclusive evidence' that rugby union players perform statistically worse in their academic achievement relative to students who had not played rugby union. ${ }^{135}$

\section{Non-contact Rugby (Touch, Tag etc)}

No relevant studies found.

\section{Wheelchair rugby}

The data from all relevant studies $(n=12)$ suggest a beneficial effect of wheelchair rugby on numerous proxy measures of well-being including quality-of-life, ${ }^{138}$ self-esteem, ${ }^{138}{ }^{139}$ selfidentity, ${ }^{139}$ functional independence, ${ }^{139}{ }^{140}$ autonomy ${ }^{141}$ and improvements in activities of daily living. ${ }^{139} 142143$

\section{Theme 5: rugby union and injuries or acute health issues}

We found 20 systematic reviews reporting on concussion and acute health issues associated with rugby union. Four of these directly concerned concussions and the remainder concerned a wide variety of injuries. Over half $(55 \%)$ of the studies directly reported on male-only cohorts, six on mixed cohorts, one on a female-only cohort and two did not directly specify the cohorts investigated. While there has been a targeted effort to standardise the definition on injuries in rugby union, ${ }^{144}$ there is still some heterogeneity which potentially distorts the true incidence, and could affect the applicability of the data.

\section{Contact rugby union (15s, 7s, etc)}

The overall incidence of injuries in senior men's professional rugby union matches was 81 per 1000 player match-hours, ${ }^{145}$ and 46.8 per 1000 player match-hours within senior male amateur rugby union players. ${ }^{146}$ The incidence of time-loss injury in male adolescent rugby union players was lower than in senior male professional players but higher than in adolescent athletes in other sports. ${ }^{147}$ Injury incidence rates in elite men's $7 \mathrm{~s}$ matches are higher than those reported for $15 \mathrm{~s}$ at the same level of competition, while injuries are also more severe. ${ }^{148}$ The injury incidence in women's $15 \mathrm{~s}$ and $7 \mathrm{~s}$ was lower than men's professional $15 \mathrm{~s}$ and $7 \mathrm{~s}$ competitions but similar to men's youth $15 \mathrm{~s}$, with a pooled incidence in women's $15 \mathrm{~s}$ of 19.6 per 1000 player match-hours. ${ }^{149}$

In regard to concussion, the results of one meta-analysis ${ }^{150}$ revealed an overall incidence in men's and women's $15 \mathrm{~s}$ of 4.73 and 0.55 per 1000 player match-hours, respectively. In men's $7 \mathrm{~s}$, concussion incidence was 3.01 per 1000 player match-hours. The incidence of concussion varied considerably between levels of play, with elite men's $15 \mathrm{~s}$ recording a rate of 0.40 concussions per 1000 player match-hours, schoolboy 15s 0.62 concussions 
Table 4 How rugby union may contribute to national and international physical activity (PA) guideline domains

PA domains

\begin{tabular}{|c|c|c|c|c|}
\hline \multirow[b]{2}{*}{ Type of rugby union } & \multicolumn{4}{|l|}{ PA domains } \\
\hline & Moderate-to-vigorous intensity PA & Non-sedentary behaviour & $\begin{array}{l}\text { Muscle/bone strengthening } \\
\text { activity }\end{array}$ & Balance-enhancing activity \\
\hline Contact rugby union & Yes & Yes & Research gap & Research gap \\
\hline $\begin{array}{l}\text { Non-contact (touch/ tag) } \\
\text { rugby }\end{array}$ & Yes & Yes & Yes (increase in lean mass) & Research gap \\
\hline Wheelchair rugby & Yes & Yes & Yes (increase in lean mass) & Research gap \\
\hline
\end{tabular}

per 1000 player match-hours (consistent with data reported elsewhere $\left.{ }^{151}\right)$, and men's community or subelite $15 \mathrm{~s}$ recording a rate of 2.08 concussions per 1000 player match-hours. The concussion risk in rugby union was reported as higher relative to other sports in two systematic reviews; ${ }^{152}{ }^{153}$ however, the authors of one suggest that this may be attributable in some degree to continuing efforts to increase awareness and implement strict concussion policies regarding reporting and removal from play. ${ }^{153}$

Non-contact Rugby (Touch, Tag etc)

No relevant studies found

\section{Wheelchair rugby}

We found one systematic review relating to injury profile in wheelchair rugby, which investigated the acute and chronic musculoskeletal injury burden of para sport. ${ }^{154}$ It reports that wheelchair rugby is one of the 'highest risk sports' for upper limb injuries.

\section{DISCUSSION}

We aimed to identify the relationships between rugby union and various health and well-being outcomes, as well as identify priorities for further research in the area. This is the first scoping review of rugby union and health and well-being, and as such comparisons are limited. However, the findings of this review are in keeping with the findings of a scoping review on golf and health, ${ }^{13}$ in that the available evidence suggests rugby union can provide health-enhancing MVPA. Specific risks associated with rugby include musculoskeletal injury and concussion, which appear to be highest in the professional game. This review also supports previously published reviews that associate sport with a range of health and well-being benefits. ${ }^{79}$

\section{Strengths and limitations}

This scoping review provides an appropriate broad overview of the research area, but a number of potential limitations may affect its applicability. While we tried modelling the review on the PRISMA-ScR checklist, ${ }^{20}$ some of the suggested steps were not feasible within the context of this review. Given the vast number of studies identified by the search strategy, and the timeline and capacity of the author group, we were unable to ensure that two of the authors independently screened $100 \%$ of the potentially relevant studies and subsequently extract $100 \%$ of the data from the included studies. Similarly, while we tried using broad search terms in the search strategy across all databases to capture all relevant studies (especially relating to well-being), we realise that our search terms may have led to us overlooking studies using more subtle psychosocial outcome measures. While we feel that our methods enabled us to find these elsewhere (eg, in the reference lists of other studies), this is something we feel needs raising.
Scoping reviews do not formally assess the methodological quality of individual studies, though we have tried to highlight significant or recurrent methodological issues encountered when critiquing individual or groups of studies. Significant variety exists as to the scientific rigour and methodological quality of individual studies, especially in relation to cross-sectional studies where populations are sometimes poorly defined, and it is not always clear if control groups have been appropriately matched. Similarly, many of the studies have a small sample size, introducing potential error in interpretation. Another limitation that needs considering in regard to injury data is that we did not have the capacity to formally critically appraise the individual studies that make up the systematic reviews, and as such the data should be interpreted in this context.

Despite these limitations, our chosen approach has enabled us to not only address the primary research questions identified in the protocol, but also to summarise the evidence in a format that is useful for policy-makers and other relevant stakeholders.

\section{Box 1 Relevant research priorities identified}

- To expand understanding of the physical activity (PA) profile of various forms of rugby union, especially in women's, youth, amateur and veteran cohorts.

- To establish the contribution of rugby union to muscle strength, balance and coordination.

- To establish the PA profile of other groups involved with the game (eg, referees, coaches, supporters).

- To establish current drivers, barriers and effective interventions for rugby union participation across the spectrum of participation (given the evidence for health benefits). This should take into account the views of multiple stakeholders (players, parents, schools, healthcare professionals), especially in non-contact and wheelchair forms of the game.

- To establish the short-term and long-term effect of rugby union participation on a range of physical health domains (e.g. neurocognitive health and function), especially in contact rugby union among amateur and women's players.

- To establish the potential effect of various forms of rugby union on mental health and well-being (especially psychosocial) markers, in particular among amateur, women's and non-contact players.

- To establish the injury profile of non-contact and wheelchair rugby, as well as continue with current injury surveillance efforts in women's and amateur contact rugby union.

- To broadly compare the health and well-being outcomes associated with rugby union, to other sports or physical activities. 


\section{What is already known?}

- Scoping reviews provide a useful framework to summarise information on a broad topic.

- Rugby union is a worldwide sport with a diverse participant base.

\section{What are the new findings?}

- All forms of rugby union (contact, non-contact, wheelchair) could be considered as evidence-based ways of providing health-enhancing moderate-to-vigorous physical activity, which is linked to a wide range of health and well-being benefits.

- More research is needed as to the relationship between contact rugby union and physical health, but there is strong evidence showing a wide range of physical health benefits for non-contact forms of rugby and wheelchair rugby in particular.

- There is a generally positive relationship between most forms of rugby union and both mental health and well-being, especially in wheelchair rugby, though further research is required outside of the wheelchair rugby setting.

- Across the spectrum of participation, contact rugby union has high injury and concussion incidence rates relative to other sports, highest at the professional level.

\section{CONCLUSIONS AND IMPLICATIONS FOR:}

\section{Existing/potential participants}

Given the findings that rugby union can provide health-enhancing PA, participants could benefit from a range of associated benefits, including a lower risk of developing type-2 diabetes mellitus, various forms of cancer, ${ }^{3155} 156$ stroke and heart disease, ${ }^{3}$ as well as a lower risk of depression. ${ }^{65} 157158$

To enable comparison against national and international guidelines for PA, ${ }^{10} 65154$ table 4 summarises how rugby union may relate to the four PA-related domains that comprise these guidelines. Further high-quality research is required to be able to concretely determine rugby union's exact contributions to these PA domains, however, especially in regard to less 'traditional' playing populations across more diverse contexts and countries.

There is evidence to support a positive relationship between both non-contact and wheelchair rugby in particular and physical health outcomes across a wide range of domains. As such, these forms of the game could be actively advocated as healthpromoting activities across the general population. The evidence base is generally mixed or less clear for contact forms of rugby union.

Despite relatively fewer studies, the relationship between rugby union and both mental health and well-being is generally positive, especially in non-professional settings. Wheelchair rugby in particular has strong wide-ranging mental health and well-being benefits.

Contact rugby union has high injury and concussion incidence rates relative to other sports across the spectrum of participation.

\section{Industry and policy-makers}

Industry and policy-makers can use the findings of this scoping review to inform current as well as future participants (and associated stakeholder groups such as parents and teachers) about the association between rugby union, and health and well-being.
Outside of wheelchair rugby (which has a generally strong supporting evidence base), there is stronger (though still limited) evidence to suggest a beneficial association between non-contact forms of rugby union and health and well-being, at a reduced 'injury cost' across the spectrum of participation relative to contact forms of the game. From a population health perspective as such, industry and policy-makers could look to further embrace elements of these formats across the playing population to potentially increase participation (especially if current injury rates are deemed as a significant barrier by some stakeholders). While gaps in the research exist that preclude any insight into the potential effect of such a shift, it could be considered a lowrisk approach to take given the current climate and conversation around contact/collision sports.

\section{Researchers}

This study has identified several gaps in the available literature (box 1). Addressing these research priorities would substantially further understanding of the relationship between rugby union, and health and well-being.

Correction notice This article has been corrected since it published Online First. The second author's name has been corrected.

Twitter Steffan A Griffin @SteffanGriffin, Nirmala Kanthi Panagodage Perera @nim_perera, Andrew Murray @docandrewmurray, Simon PT Kemp @drsimonkemp, Keith A Stokes@drkeithstokes and Paul Kelly @narrowboat_paul

Contributors SAG proposed the review. SAG, AM, KAS and SK identified the method and identified the framework for the review. SAG, AM, NPP and CH proposed the search strategy. All authors contributed to the development of the research questions and the study design. All authors developed the first and subsequent drafts of the manuscript, then subsequently reviewed and approved the final manuscript.

Funding This project formed part of SAG's work with the Department of Primary Care and Public Health at Imperial College London, a remunerated post, as well as SAG's work as a Sports Medicine Training Fellow at the Rugby Football Union. NPP is funded from the Centre for Sport, Exercise and Osteoarthritis Research Versus Arthritis (sports grant number: HFR02510).

Competing interests SAG receives remuneration for clinical work in professional rugby union, and is employed by the Rugby Football Union as a Sports Medicine Training Fellow. AM receives remuneration from Scottish Rugby Union for clinical work. SK is employed by the Rugby Football Union as medical services director. KAS is employed by the Rugby Football Union as medical research lead.

\section{Patient consent for publication Not required.}

Provenance and peer review Not commissioned; externally peer reviewed.

Supplemental material This content has been supplied by the author(s). It has not been vetted by BMJ Publishing Group Limited (BMJ) and may not have been peer-reviewed. Any opinions or recommendations discussed are solely those of the author(s) and are not endorsed by BMJ. BMJ disclaims all liability and responsibility arising from any reliance placed on the content. Where the content includes any translated material, BMJ does not warrant the accuracy and reliability of the translations (including but not limited to local regulations, clinical guidelines, terminology, drug names and drug dosages), and is not responsible for any error and/or omissions arising from translation and adaptation or otherwise.

\section{ORCID iDs}

Steffan A Griffin http://orcid.org/0000-0002-5493-0911

Nirmala Kanthi Panagodage Perera http://orcid.org/0000-0001-6110-8945

Andrew Murray http://orcid.org/0000-0003-3327-8028

Catherine Hartley http://orcid.org/0000-0002-3495-1053

Simon P T Kemp http://orcid.org/0000-0002-3250-2713

Keith A Stokes http://orcid.org/0000-0002-5049-2838

Paul Kelly http://orcid.org/0000-0003-1946-9848

\section{REFERENCES}

1 World Rugby. Player numbers, 2017. Available: https://www.world.rugby/ development/player-numbers?lang=en [Accessed Dec 2019].

2 Moy K, Scragg R, McLean G, et al. Metabolic equivalent (Met) intensities of culturally-specific physical activities performed by new Zealanders. N Z Med J 2006;119:6. 
3 Lee I-M, Shiroma EJ, Lobelo F, et al. Effect of physical inactivity on major noncommunicable diseases worldwide: an analysis of burden of disease and life expectancy. Lancet 2012;380:219-29.

4 Arem $\mathrm{H}$, Moore SC, Patel A, et al. Leisure time physical activity and mortality: a detailed pooled analysis of the dose-response relationship. JAMA Intern Med 2015;175:959-67.

5 Ford ES, Caspersen CJ. Sedentary behaviour and cardiovascular disease: a review of prospective studies. Int J Epidemiol 2012;41:1338-53.

6 Proper KI, Singh AS, van Mechelen W, et al. Sedentary behaviors and health outcomes among adults: a systematic review of prospective studies. Am J Prev Med 2011:40:174-82

7 Oja P, Titze S, Kokko S, et al. Health benefits of different sport disciplines for adults: systematic review of observational and intervention studies with meta-analysis. $\mathrm{Br} \mathrm{J}$ Sports Med 2015;49:434-40.

8 Khan KM, Thompson AM, Blair SN, et al. Sport and exercise as contributors to the health of nations. The Lancet 2012;380:59-64

9 Oja P, Kelly P, Pedisic Z, et al. Associations of specific types of sports and exercise with all-cause and cardiovascular-disease mortality: a cohort study of 80306 British adults. Br J Sports Med 2017:51:812-7.

10 World Health Organisation. More active people for a healthier world. the global action plan on physical activity. World Health Organisaion, 2018.

11 World Health Organisation. Preamble to the constitution of the world health organisation as adopted by the International health conference. World Health Organisation, 1946

12 Affuso 0, Cox TL, Durant NH, et al. Attitudes and beliefs associated with leisure-time physical activity among African American adults. Ethn Dis 2011;21:63-7.

13 Murray AD, Daines L, Archibald D, et al. The relationships between golf and health: a scoping review. Br J Sports Med 2017;51:12-19.

14 Grant MJ, Booth A. A typology of reviews: an analysis of 14 review types and associated methodologies. Health Info Libr J 2009:26:91-108.

15 Arksey H, O’Malley L. Scoping studies: towards a methodological framework. Int J Soc Res Methodol 2005;8:19-32.

16 Levac D, Colquhoun H, O'Brien KK. Scoping studies: advancing the methodology. Implementation Science 2010;5:69.

17 Peters MDJ, Godfrey CM, Khalil H, et al. Guidance for conducting systematic scoping reviews. Int J Evid Based Healthc 2015;13:141-6.

18 Murray A, Daines L, Archibald D, et al. The relationship and effects of golf on physical and mental health: a scoping review protocol. Br J Sports Med 2016;50:647-50.

19 Griffin SA, Panagodage Perera NK, Murray A, et al. The relationships between rugby Union and health: a scoping review protocol. BMJ Open Sport Exerc Med 2019:5:e000593.

20 Tricco AC, Lillie E, Zarin W, et al. PRISMA extension for scoping reviews (PRISMAScR): checklist and explanation. Ann Intern Med 2018;169:467-73.

21 Kelly P, Williamson C, Niven AG, et al. Walking on sunshine: Scoping review of the evidence for walking and mental health. Br J Sports Med 2018;52:800-6.

22 Braun V, Clarke V. Using thematic analysis in psychology. Qual Res Psychol 2006;3:77-101.

23 Bree RT, Gallagher G. Using Microsoft Excel to code and thematically analyse qualitative data: a simple, cost-effective approach. AISHE-J 2016;8.

24 Rugby A. Asia Rugby drives record-breaking year for rugby's global growth. Asia Rugby, 2019. Available: https://www.asiarugby.com/2019/07/10/rugbys-globalgrowth/ [Accessed December 2019].

25 Beaven RP, Highton JM, Thorpe M-C, et al. Movement and physiological demands of international and regional Men's touch rugby matches. J Strength Cond Res 2014:28:3274-9.

26 Dubois R, Paillard T, Lyons M, et al. Running and metabolic demands of elite rugby Union assessed using traditional, metabolic power, and heart rate monitoring methods. J Sports Sci Med 2017;16:84-92.

27 Cunniffe B, Proctor W, Baker JS, et al. An evaluation of the physiological demands of elite rugby Union using global positioning system tracking software. J Strength Cond Res 2009;23:1195-203.

28 Suarez-Arrones L, Portillo J, Pareja-Blanco F, et al. Match-play activity profile in elite women's rugby union players. J Strength Cond Res 2014;28:452-8.

29 LMT V, Goncalves BSV, Figueira BEN, et al. Influence of different small-sided games on physical and physiological demands in rugby union players. International Journal of Sports Science \& Coaching 2016;11:78-84.

30 Patience MA, Kilpatrick MW, Sun H, et al. Sports game play: a comparison of moderate to vigorous physical activities in adolescents. J Sch Health 2013;83:818-23

31 Hogarth LW, Burkett BJ, McKean MR. Activity profiles and physiological responses of representative tag football players in relation to playing position and physical fitness. PLoS One 2015;10:e0144554.

32 Borel B, Lacroix J, Daviet J-C, et al. Intensity level and on-court role of wheelchair rugby players during competition. J Sports Med Phys Fitness 2019;59:387-93.

33 Hör G, Louit L, Coste N, et al. Exercise intensity during wheelchair rugby competition. Ann Phys Rehabil Med 2012;55:e251-51.

34 Roberts SP, Trewartha G, Higgitt RJ, et al. The physical demands of elite English rugby Union. J Sports Sci 2008;26:825-33.
35 Virr JL, Game A, Bell GJ, et al. Physiological demands of women's rugby union: timemotion analysis and heart rate response. J Sports Sci 2014:32:239-47.

36 Venter RE, Opperman E, Opperman S. The use of global positioning system (GPs) tracking devices to assess movement demands and impacts in Under-19 rugby Union match play. Af. J. Phys, Health Edu. Rec. \& Dance 2011;17:1-8.

37 Tee JC, Lambert MI, Coopoo Y. Gps comparison of training activities and game demands of professional rugby Union. Int J Sports Sci Coach 2016;11:200-11.

38 Ross A, Gill N, Cronin J. The match demands of international rugby sevens. J Sports Sci 2015;33:1035-41

39 Ross A, Gill ND, Cronin JB. A comparison of the match demands of international and provincial rugby Sevens. Int J Sports Physiol Perform 2015;10:786-90.

40 Rienzi E, Reilly T, Malkin C. Investigation of anthropometric and work-rate profiles of rugby Sevens players. Journal of Sports Medicine \& Physical Fitness 1999;39:160-4

41 Read DB, Jones B, Williams S, et al. The physical characteristics of specific phases of play during rugby Union match play. Int I Sports Physiol Perform 2018;13:1331-6.

42 Read D, Weaving D, Phibbs P, et al. Movement and physical demands of school and university rugby Union match-play in England. BMJ Open Sport Exerc Med 2016:2:e000147.

43 Quarrie KL, Hopkins WG, Anthony MJ, et al. Positional demands of international rugby Union: evaluation of player actions and movements. J Sci Med Sport 2013;16:353-9.

44 McLean DA. Analysis of the physical demands of international rugby Union. J Sports Sci 1992; 10:285-96.

45 Jones MR, West DJ, Crewther BT, et al. Quantifying positional and temporal movement patterns in professional rugby Union using global positioning system. Eur J Sport Sci 2015;15:488-96.

46 Hartwig TB, Naughton G, Searl J. Motion analyses of adolescent rugby union players: a comparison of training and game demands. Journal of Strength and Conditioning Research 2011;25:966-72.

47 Cunningham DJ, Shearer DA, Drawer S, et al. Movement demands of elite Under-20s and senior international rugby union players. PLoS One 2016;11:e0164990.

48 Coughlan GF, Green BS, Pook PT, et al. Physical game demands in elite rugby Union: a global positioning system analysis and possible implications for rehabilitation. J Orthop Sports Phys Ther 2011;41:600-5.

49 Couderc A, Gabbett TJ, Piscione J, et al. Repeated high-intensity effort activity in international male rugby Sevens. J Strength Cond Res 2019;22:22.

50 Couderc $A$, Thomas $C$, Lacome $M$, et al. Movement patterns and metabolic responses during an international rugby Sevens tournament. Int I Sports Physiol Perform 2017:12:901-7.

51 Clarke AC, Anson JM, Pyne DB. Game movement demands and physical profiles of junior, senior and elite male and female rugby sevens players. J Sports $\mathrm{SCi}$ 2017:35:727-33.

52 Clarke AC, Anson J, Pyne D. Physiologically based GPS speed zones for evaluating running demands in Women's Rugby Sevens. J Sports Sci 2015:33:1101-8.

53 Cahill N, Lamb K, Worsfold P, et al. The movement characteristics of English Premiership rugby union players. J Sports Sci 2013:31:229-37.

54 Beard A, Chambers R, Millet GP, et al. Comparison of game movement positional profiles between professional Club and senior international rugby union players. Int Sports Med 2019;40:385-9.

55 Austin D, Gabbett T, Jenkins D. The physical demands of super 14 rugby Union. J Sci Med Sport 2011:14:259-63.

56 Pollard BT, Turner AN, Eager R, et al. The ball in play demands of international rugby Union. J Sci Med Sport 2018:21:1090-4.

57 Lacome M, Piscione J, Hager J-P, et al. A new approach to quantifying physical demand in rugby Union. J Sports Sci 2014;32:290-300.

58 Vescovi JD, Goodale T. Physical demands of women's rugby Sevens matches: female athletes in motion (FAiM) study. Int J Sports Med 2015:36:887-92.

59 Suarez-Arrones L, Portillo LJ, García JM, et al. Running demands and heart rate response in rugby Union Referees. J Strength Cond Res 2013;27:2946-51.

60 Suarez-Arrones L, Nuñez FJ, Portillo J, et al. Match running performance and exercise intensity in elite female rugby Sevens. J Strength Cond Res 2012;26:1858-62.

61 Malone S, Earls M, Shovlin A, et al. Match-Play Running Performance and Exercise Intensity in Elite International Women's Rugby Sevens. Journal of Strength \& Conditioning Research 2018:14:14.

62 Cunniffe B, Proctor W, Baker JS, et al. An evaluation of the physiological demands of elite rugby Union using global positioning system tracking software. J Strength Cond Res 2009;23:1195-203.

63 Goodale TL, Gabbett TJ, Tsai M-C, et al. The Effect of Contextual Factors on Physiological and Activity Profiles in International Women's Rugby Sevens. Int J Sports Physiol Perform 2017:12:370-6.

64 National Centre for Social Research. Health survey for England, 2016. Health and Social Care Information Centre, 2017.

65 Public Health England. Everybody active, every day: framework for physical activity. Public Health England, 2018.

66 Australian Bureay of Statistics. National health survey: first results, 2017-18. Australian Bureau of Statistics, 2019 
67 Foster C, Armstrong MEG. What types of physical activities are effective in developing muscle and bone strength and balance? Journal of Frailty, Sarcopenia and Falls 2018;03:58-65.

68 Strain T, Fitzsimons C, Kelly P, et al. The forgotten guidelines: cross-sectional analysis of participation in muscle strengthening and balance \& co-ordination activities by adults and older adults in Scotland. BMC Public Health 2016;16:1108.

69 Davies MAM, D Judge A, Delmestri A, et al. Health amongst former rugby union players: a cross-sectional study of morbidity and health-related quality of life. Sci Rep 2017;7:11786.

70 McMillan TM, McSkimming P, Wainman-Lefley J, et al. Long-Term health outcomes after exposure to repeated concussion in elite level: rugby union players. I Neurol Neurosurg Psychiatry 2017;88:505-11.

71 Climstein M, Walsh J, Best J, et al. Demographics of veteran rugby Union competitors. J Sci Med Sport 2011;14:e80-1.

72 Mike C, Joe W, Best J, et al. Physiological and pathology demographics of veteran rugby athletes: golden oldies rugby festival. World Academy of Science, Engineering and Technology, International Journal of Medical, Health, Biomedical, Bioengineering and Pharmaceutical Engineering 2011;5:227-31.

73 Walsh J, Climstein M, Burke S, et al. Medical and health histories of golden oldies world rugby festival participants. Football Science 2011;8:230.

74 Hübner-Woźniak E, Morgulec-Adamiec N, Malara M, et al. Effect of training on the serum lipid profile in able-bodied and spinal cord injured rugby players. Bio/ Sport 2010;27:269-72.

75 Haluzík M, Boudová L, Nedvídková J, et al. Lower serum leptin concentrations in rugby players in comparison with healthy non-sporting subjects--relationships to anthropometric and biochemical parameters. Eur J Appl Physiol Occup Physiol 1998;79:58-61.

76 Evelson P, Gambino G, Travacio M, et al. Higher antioxidant defences in plasma and low density lipoproteins from rugby players. Eur J Clin Invest 2002;32:818-25.

77 Imamura H, lide K, Yoshimura Y, et al. Nutrient intake, serum lipids and iron status of colligiate rugby players. J Int Soc Sports Nutr 2013;10:9.

78 Huang S, Wei C, Tung I. The aging effect on the ratio of total cholesterol to high density lipoprotein between national rugby players and untrained males.7th world Congress on aging and physical activity. Journal of Aging \& Physical Activity 2008; 16:S46-S

79 Davies MA, Judge A, Delmestri A, et al. Joint replacement and health-related quality of life amongst former rugby players. Osteoarthritis Cartilage 2017:25:S195.

80 Davies M, Judge A, Stokes K, et al. Is rugby playing load predictive of lower limb osteoarthritis in former international rugby players? Osteoarthritis Cartilage 2016;24:S533-4.

81 Burgess L, Gavin J. The incidence of injuries and epidemiology of osteoarthritis in retired, amateur, rugby union players. Lancaster: Poster at the Research and Impact in Active Ageing Conference, 2017June.

82 Egan E, Reilly T, Giacomoni M, et al. Bone mineral density among female sports participants. Bone 2006;38:227-33.

83 Elloumi M, Ounis OB, Courteix D, et al. Long-Term rugby practice enhances bone mass and metabolism in relation with physical fitness and playing position. J Bone Miner Metab 2009;27:713-20.

84 Elloumi M, Courteix D, Sellami S, et al. Bone mineral content and density of Tunisian male rugby players: differences between forwards and backs. Int I Sports Med 2006;27:351-8.

85 Elloumi M, Courteix D, Zaouali M, et al. Rugby practice and skeleton: correlations between strength, bone mineral density, and biochemical markers of bone remodeling. Sci Sports 2006;21:96-8.

86 Ginty F, Rennie KL, Mills L, et al. Positive, site-specific associations between bone mineral status, fitness, and time spent at high-impact activities in 16- to 18-year-old boys. Bone 2005:36:101-10.

87 Gorla Jl, Costa e Silva AdeA, Borges M, et al. Impact of wheelchair rugby on body composition of subjects with tetraplegia: a pilot study. Arch Phys Med Rehabil 2016;97:92-6.

88 Nevill AM, Holder RL, Stewart AD. Do sporting activities convey benefits to bone mass throughout the skeleton? J Sports Sci 2004;22:645-50.

89 Zakhem E, El Khoury G, Zouhal H, et al. An observational study on the impact of physical activity type on bone mineral density, hip bone geometry and tbs in a group of adult men. Kinesitherapie 2015;15:24-9.

90 Hardcastle SA, Dieppe P, Gregson CL, et al. Osteoarthritis and bone mineral density: are strong bones bad for joints? Bonekey Rep 2015;4

91 Berge J, Marque B, Vital J-M, et al. Age-Related changes in the cervical spines of front-line rugby players. Am J Sports Med 1999;27:422-9.

92 Brauge D, Delpierre C, Adam P, et al. Incidence of cervical spine degenerative complications in former professional rugby players. Eur Spine J 2013;22:1212.

93 Brauge D, Delpierre C, Adam P, et al. Clinical and radiological cervical spine evaluation in retired professional rugby players. J Neurosurg 2015;23:551-7.

94 Hogan BA, Hogan NA, Vos PM, et al. The cervical spine of professional front-row rugby players: correlation between degenerative changes and symptoms. Ir J Med Sci 2010;179:259-63.

95 Scher AT. Premature onset of degenerative disease of the cervical spine in rugby players. S Afr Med J 1990;77:557-8.
96 Cunningham J, Broglio S, Wilson F. Influence of playing rugby on long-term brain health following retirement: a systematic review and narrative synthesis. BMJ Open Sport Exerc Med 2018;4:e000356.

97 Alexander D, Shuttleworth-Edwards A, Kidd M, et al. Concussions in youth rugby: a prospective investigation of enduring neurocognitive and academic effects on players vs non-contact sports controls. Brain Inj 2016;30:483-4.

98 Alexander DG, Shuttleworth-Edwards AB, Kidd M, et al. Mild traumatic brain injuries in early adolescent rugby players: long-term neurocognitive and academic outcomes. Brain Inj 2015;29:1113-25

99 Gardner A, Shores EA, Batchelor J. Reduced processing speed in rugby union players reporting three or more previous concussions. Arch Clin Neuropsychol 2010;25:174-81.

100 Shuttleworth-Edwards AB, Smith I, Radloff SE. Neurocognitive vulnerability amongst university rugby players versus noncontact sport controls. J Clin Exp Neuropsychol 2008;30:870-84.

101 Basson WJ, Essack FA. Neurobehavioural profile following cumulative mild traumatic brain injury in adolescent rugby players in South Africa. Journal of Psychology in Africa 2017;27:553-6.

102 Owens TS, Calverley TA, Stacey BS, et al. Elevated Systemic Oxidative-Nitrosative Stress and Cerebrovascular Function in Professional Rugby Union Players: The Link to Impaired Cognition. London: Poster at the Europhysiology conference, 2018

103 Pentz HL. Do cumulative mild head injuries in rugby affect neuropsychological performance?: a comparative study between Club rugby players and non-contact sport athletes. Pietermaritzburg: University of KwaZulu-Natal, 2008.

104 Ancer RL. Cumulative mild head injury in rugby: cognitive test profiles of professional rugby and cricket players. PHD thesis. Rhodes University, 1999.

105 Beilinsohn T. Cumulative mild head injury in rugby: a comparison of cognitive deficit and Postconcussive symptomatology between Schoolboy rugby players and noncontact sport controls. PHD thesis. Rhodes University, 2001

106 Decq P, Gault N, Blandeau M, et al. Long-Term consequences of recurrent sports concussion. Acta Neurochir 2016;158:289-300.10.1007/s00701-015-2681-4

107 Hunt TN, LeMay D. Normal Cognitive Function Following Self-reported "Dings" In A Cohort Of Collegiate Rugby Players. Medicine and Science in Sports and Exercise 2012:44:411.

108 Lefebvre G, Chamard E, Proulx S, et al. Increased myo-inositol in primary motor cortex of contact sports athletes without a history of concussion. J Neurotrauma 2018;35:953-62.

109 Rapetsoa MJ. Cumulative mild head injury (CMHI) in contact sports: an evaluation of pre and post season cognitive profiles rugby players compared with non-contact sport controls at the University of Limpopo (Turfloop campus). PHD thesis. University of Limpopo, 2015.

110 Barton W, Penney NC, Cronin O, et al. The microbiome of professional athletes differs from that of more sedentary subjects in composition and particularly at the functional metabolic level. Gut 2018;67:625-33.

111 Lindsay A, Lewis J, Gill N, et al. Immunity, inflammatory and psychophysiological stress response during a competition of professional rugby Union. Pteridines 2015:26:153-60.

112 Filliau C, Younes M, Blanchard A-L, et al. Effect of "Touch Rugby" Training on the Cardiovascular Autonomic Control In Sedentary Subjects. Int I Sports Med 2015:36:567-72

113 Ashton R. A quantitative and qualitative evaluation of a 10 week play touch rugby league programme for improving physical activity in adult men and women. Master's Thesis. United Kingdom: University of Chester, 2015.

114 Dobbin N. The role of 'Play Touch Rugby League'and self-paced interval running for improving men's health. Master's Thesis. United Kingdom: University of Chester 2015.

115 Mendham AE, Duffield R, Marino F, et al. Small-sided games training reduces CRP, IL-6 and leptin in sedentary, middle-aged men. Eur J App/ Physiol 2014;114:2289-97.

116 Mendham AE, Duffield R, Coutts AJ, et al. Rugby-specific small-sided games training is an effective alternative to stationary cycling at reducing clinical risk factors associated with the development of type 2 diabetes: a randomized, controlled trial. PLoS One 2015;10:e0127548.

117 Mendham AE, Coutts AJ, Duffield R. The acute effects of aerobic exercise and modified rugby on inflammation and glucose homeostasis within Indigenous Australians. Eur J Appl Physiol 2012;112:3787-95.

118 Calcaterra V, Larizza D, Codrons E, et al. Improved metabolic and cardiorespiratory fitness during a recreational training program in obese children. $J$ Pediatr Endocrinol Metab 2013:26:271-6.

119 Mendham AE, Duffield R, Marino F, et al. Differences in the acute inflammatory and glucose regulatory responses between small-sided games and cycling in sedentary, middle-aged men. J Sci Med Sport 2015;18:714-9.

120 Domaszewska K, Ł S. Spirometric and ergospirometric evaluation of wheelchair rugby players. Trends in Sport Sciences 2013;20:89-94.

121 Matos-Souza JR, de Rossi G, Costa E Silva AA, et al. Impact of adapted sports activities on the progression of carotid atherosclerosis in subjects with spinal cord injury. Arch Phys Med Rehabil 2016;97:1034-7. 
122 Phillips A, Squair JW, Currie K, et al. Parapan American games: does physical activity participation improve cerebrovascular function after high-level spinal cord injury? FASEB Journal Conference: Experimental Biology 2015;2016:30.

123 Sarro KJ, Paris JV, Moreno MA, et al. Thoracoabdominal mobility is improved in subjects with tetraplegia after one year of wheelchair rugby training. Sci Sports 2016;31:261-9.

124 Moreno MA, Zamunér AR, Paris JV, et al. Effects of wheelchair sports on respiratory muscle strength and thoracic mobility of individuals with spinal cord injury. Am J Phys Med Rehabil 2012;91:470-7.

125 Moreno MA, Paris JV, Sarro KJ, et al. Wheelchair rugby improves pulmonary function in people with tetraplegia after 1 year of training. J Strength Cond Res 2013:27:50-6.

126 Gouttebarge V, Aoki H, Lambert M, et al. A history of concussions is associated with symptoms of common mental disorders in former male professional athletes across a range of sports. Phys Sportsmed 2017;45:443-9.

127 Gouttebarge V, Kerkhoffs G, Lambert M. Prevalence and determinants of symptoms of common mental disorders in retired professional rugby union players. Eur J Sport Sci 2016;16:595-602.

128 Gouttebarge V, Hopley P, Kerkhoffs G, et al. Symptoms of common mental disorders in professional rugby: an international observational descriptive study. Int J Sports Med 2017;38:864-70.

129 Gouttebarge V, Hopley P, Kerkhoffs G, et al. A 12-month prospective cohort study of symptoms of common mental disorders among professional rugby players. Eur J Sport Sci 2018;18:1004-12.

130 Silveira SL, Ledoux T, Cottingham M, et al. Association among practice frequency on depression and stress among competitive US male wheelchair rugby athletes with tetraplegia. Spinal Cord 2017;55:957-62.

131 Cheng Eva (Hui-Ping), Pegg S, Stebbins R. Old bodies, young hearts: a qualitative exploration of the engagement of older male amateur rugby union players in Taiwan. Leisure Studies 2016;35:549-63.

132 Dong E, Zhang L, Choe J, et al. Rugby Union among middle-aged American men: an exploration. Leisure Studies 2013;32:219-28.

133 Fields SK, Comstock RD. Why American women play rugby. Women in Sport Physical Activity Journal 2008;17:8-18.

134 Kriel MG. Academic achievement in early adolescent rugby players with multiple concussions: a retrospective analysis. Master's Thesis. North-West University, 2012.

135 Bradley J, Keane F, Crawford S. School sport and academic achievement. J Sch Health 2013;83:8-13.

136 Laubscher JA, Dijkstra HP, Strydom GL, et al. Academic consequences of very mild and mild traumatic brain injuries in secondary school rugby players. Af. J. Phys, Health Edu. Rec. \& Dance 2010;16:221-30.

137 Côté-Leclerc F, Boileau Duchesne G, Bolduc P, et al. How does playing adapted sports affect quality of life of people with mobility limitations? results from a mixedmethod sequential explanatory study. Health Qual Life Outcomes 2017;15:22.

138 Zelenka T, Kudláček M, Wittmannová J. Quality of life of wheelchair rugby players. European Journal of Adapted Physical Activity 2017;10:31-5.
139 Furmaniuk L, Cywińska-Wasilewska G, Kaczmarek D. Influence of long-term wheelchair rugby training on the functional abilities in persons with tetraplegia over a two-year post-spinal cord injury. J Rehabil Med 2010;42:688-90.

140 Haslett D, Fitzpatrick B, Breslin G. The psychological influences on participation in wheelchair rugby: a social relational model of disability. Auc Kinanthropologica 2017:53:60-78.

141 Lewandowski A, Piekorz Z, Srokowska A, et al. Rugby and physical fitness of people with tetraplegia. Journal of Health Sciences 2013;3:372-83.

142 Adnan Y, McKenzie A, Miyahara M. Self-Efficacy for quad rugby skills and activities of daily living. Adapt Phys Act Q 2001;18:90-101.

143 Fuller CW, Molloy MG, Bagate C, et al. Consensus statement on injury definitions and data collection procedures for studies of injuries in rugby Union. Br J Sports Med 2007:41:328-31.

144 Williams S, Trewartha G, Kemp S, et al. A meta-analysis of injuries in senior men's professional rugby Union. Sports Med 2013;43:1043-55.

145 Yeomans C, Kenny IC, Cahalan R, et al. The incidence of injury in amateur male rugby Union: a systematic review and meta-analysis. Sports Med 2018;48:837-48.

146 Bleakley C, Tully M, O'Connor S. Epidemiology of adolescent rugby injuries: a systematic review. J Athl Train 2011;46:555-65.

147 Cruz-Ferreira A, Cruz-Ferreira E, Santiago L, et al. Epidemiology of injuries in senior male rugby Union sevens: a systematic review. Phys Sportsmed 2017:45:41-8.

148 King D, Hume P, Cummins C, et al. Match and Training Injuries in Women's Rugby Union: A Systematic Review of Published Studies. Sports Med 2019:1-16.

149 Gardner AJ, Iverson GL, Williams WH, et al. A systematic review and meta-analysis of concussion in rugby Union. Sports Med 2014;44:1717-31.

150 Kirkwood G, Parekh N, Ofori-Asenso R, et al. Concussion in youth rugby Union and rugby League: a systematic review. Br J Sports Med 2015;49:506-10.

151 Pfister T, Pfister K, Hagel B, et al. The incidence of concussion in youth sports: a systematic review and meta-analysis. Br J Sports Med 2016;50:292-7.

152 Prien A, Grafe A, Rössler R, et al. Epidemiology of head injuries focusing on concussions in team contact sports: a systematic review. Sports Med 2018;48:953-69.

153 Ottesen T, Mashikovskiy E, Gentry M, et al. Acute and chronic musculoskeletal injury in para sport: a systematic review. Clin J Sport Med 2018;28:227.

154 Department of Health and Social Care. Uk chief medical officers' physical activity guidelines. Department of Health and Social Care, 2019.

155 Department of Health. Start active, stay active: A report on physical activity from the four home countries' Chief Medical Officers. Department of Health, 2011.

156 Lincoln P, Fenton K, Alessi C, et al. The Blackfriars consensus on brain health and dementia. The Lancet 2014;383:1805-6.

157 Weyerer S. Physical inactivity and depression in the community. Int J Sports Med 1992;13:492-6.

158 Schuch FB, Vancampfort D, Firth J, et al. Physical activity and incident depression: a meta-analysis of prospective cohort studies. Am J Psychiatry 2018:175:631-48. 